Cahiers $d u$ MONDE RUSSE

\section{Cahiers du monde russe}

Russie - Empire russe - Union soviétique et États indépendants

$52 / 4 \mid 2011$

Varia

\title{
Kevin M.F. Platt, Terror and Greatness
}

\section{Mihail Veližev}

\section{OpenEdition \\ Journals}

Édition électronique

URL : http://journals.openedition.org/monderusse/7663

DOI : $10.4000 /$ monderusse. 7663

ISSN : $1777-5388$

Éditeur

Éditions de l'EHESS

\section{Édition imprimée}

Date de publication : 20 décembre 2011

Pagination : 819-825

ISBN : 978-2-7132-2353-2

ISSN : $1252-6576$

\section{Référence électronique}

Mihail Veližev, « Kevin M.F. Platt, Terror and Greatness », Cahiers du monde russe [Онлайн], 52/4 | 2011, Выложить онлайн 03 décembre 2012, Наводить справки в 22 septembre 2020. URL : http:// journals.openedition.org/monderusse/7663; DOI : https://doi.org/10.4000/monderusse.7663

Ce document a été généré automatiquement le 22 septembre 2020

〔c École des hautes études en sciences sociales 


\title{
Kevin M.F. Platt, Terror and Greatness
}

\author{
Mihail Veližev
}

\section{RÉFÉRENCE}

Kevin M.F. PlatT, Terror and Greatness. Ivan and Peter as Russian Myths. Ithaca :

Cornell University Press, 2011, XI +294 p.

1 В 1878 Г. В ЖУРНАЛЕ «ДРЕВНЯЯ И НОВАЯ РОССИЯ» ПОЯВИЛСЯ ОЧЕРК ИЗВЕСТНОГО ПУБЛИЦИСТА, ИСТОРИКА И ЛИТЕРАТОРА Д.Л. МОРДОВЦЕВА О ЗНАМЕНИТЫХ СЫНОУБИЙЦАХ, ЧЬИ ПРЕСТУПЛЕНИЯ НОСИЛИ ОТЧЕТЛИВО «ВОЗВЫШЕННЫЙ» ХАРАКТЕР 1. В ЧИСЛЕ КЛАССИЧЕСКИХ ДЕТОУБИЙЦ ФИГУРИРОВАЛИ ТРИ В РАВНОЙ СТЕПЕНИ МИФОЛОГИЗИРОВАННЫХ ПЕРСОНАЖА: ИВАН ГРОЗНЫЙ, ПЕТР ВЕЛИКИЙ И ТАРАС БУЛЬБА. РОССИЯНИНУ ЭПОХИ НИКОЛАЯ І ТАКОЕ СРАВНЕНИЕ ПОКАЗАЛОСЬ БЫ ПОЧТИ КОЩУНСТВЕННЫМ. В ЕГО ГЛАЗАХ, ПЕТР ВЕЛИКИЙ ОЛИЦЕТВОРЯЛ ЕВРОПЕИЗИРОВАННУЮ РОССИЮ, БЫЛ ЦЕНТРАЛЬНЫМ ГЕРОЕМ ИМПЕРАТОРСКОГО «СЦЕНАРИЯ ВЛАСТИ», ТОГДА КАК ОБ ИВАНЕ ГРОЗНОМ, НАПРОТИВ, ДО СЕРЕДИНЫ ХІХ В. ВООБЩЕ БЫЛО МАЛО ЧТО ИЗВЕСТНО, А СВЕДЕНИЯ, ДОСТУПНЫЕ ОБРАЗОВАННЫМ ЛЮДЯМ ТОГО ВРЕМЕНИ БЛАГОДАРЯ «ИСТОРИИ ГОСУДАРСТВА РОССИЙСКОГО» КАРАМЗИНА, ХАРАКТЕРИЗОВАЛИ ПЕРВОГО РУССКОГО ЦАРЯ С ВЕСЬМА НЕПРИГЛЯДНОЙ СТОРОНЫ. ЧТО ЖЕ ДО ТАРАСА БУЛЬБЫ, ТО ЭТОТ ПРАВОСЛАВНЫЙ, «НАРОДНЫЙ», ГЕРОИЧЕСКИЙ И ПОТОМУ «ПРАВИЛЬНЫЙ» ПЕРСОНАЖ ИМЕЛ ЛИТЕРАТУРНУЮ ПРИРОДУ И ЕГО СРАВНЕНИЕ С РЕАЛЬНЫМИ МОНАРХАМИ НЕ МОГЛО КАЗАТЬСЯ САМО СОБОЙ РАЗУМЕЮЩИМСЯ. НАПРОТИВ, В КОНЦЕ ХІХ - НАЧАЛЕ ХХ ВВ. ЧИТАТЕЛИ МОРДОВЦЕВА ВОСПРИНЯЛИ БЫ СОПОЛОЖЕНИЕ ИВАНА, ПЕТРА И ТАРАСА БУЛЬБЫ КАК ВПОЛНЕ УМЕСТНОЕ, А ПРОТИВОПОСТАВЛЕНИЕ «ВЛАСТИ РОДИТЕЛЬСКОЙ, УСЛОЖНЕННОЙ [...] ИСТОРИЧЕСКОЙ НЕОБХОДИМОСТЬЮ» «ПРОТЕСТАМ МОЛОДОСТИ» ${ }^{2}$ - КАК НЕЧТО ЕСТЕСТВЕННОЕ. МОРДОВЦЕВ УКАЗАЛ НА ВАЖНЕЙШИЙ СДВИГ В ВОСПРИЯТИИ КЛЮЧЕВЫХ ФИГУР РУССКОГО ПОЛИТИКО-КУЛЬТУРНОГО ПАНТЕОНА, ПРОИЗОШЕДШИЙ В ТЕЧЕНИЕ ВТОРОЙ 
ПОЛОВИНЫ ХІХ СТОЛЕТИЯ. СТО ТРИДЦАТЬ ТРИ ГОДА СПУСТЯ АМЕРИКАНСКИЙ ИСТОРИК ЛИТЕРАТУРЫ КЕВИН ПЛАТТ ИССЛЕДУЕТ ПРОЦЕСС ТРАНСФОРМАЦИИ РЕПУТАЦИЙ ДВУХ РОССИЙСКИХ МОНАРХОВ В СВОЕЙ НОВОЙ КНИГЕ «УЖАС И ВЕЛИЧИЕ. ИВАН И ПЕТР КАК РУССКИЕ МИФЫ».

КНИГА ПЛАТТА ПОВЕСТВУЕТ О ФУНКЦИИ МИФОЛОГИЗИРОВАННЫХ «ИВАНА ГРОЗНОГО» И «ПЕТРА ВЕЛИКОГО» В ПРОЦЕССЕ ФОРМИРОВАНИЯ РУССКОЙ КОЛЛЕКТИВНОЙ ИДЕНТИЧНОСТИ, В КУЛЬТУРНОЙ ПАМЯТИ ОБРАЗОВАННОЙ ЭЛИТЫ (КАК СЛЕДСТВИЕ, АВТОР ОТКАЗЫВАЕТСЯ ОТ РАССМОТРЕНИЯ «НАРОДНЫХ» ОБРАЗОВ ИВАНА И ПЕТРА В ИСТОРИЧЕСКИХ ПЕСНЯХ И ФОЛЬКЛОРНЫХ ТЕКСТАХ, А ТАКЖЕ ОТ АНАЛИЗА РЕПУТАЦИИ РУССКИХ МОНАРХОВ В МАССОВОМ ИСКУССТВЕ ХХ В.). КОРПУС ИСТОЧНИКОВ, КОТОРЫЕ ИСПОЛЬЗУЕТ ПЛАТТ, ДЕЛИТСЯ НА ТРИ ЧАСТИ: ИСТОРИОГРАФИЯ, ЛИТЕРАТУРА И ИСКУССТВО (ТЕАТР, ЖИВОПИСЬ, СКУЛЬПТУРА, КИНО). МОНОГРАФИЯ СОСТОИТ ИЗ СЕРИИ case-studies, СТРОЯЩИХСЯ ВОКРУГ АВТОРИТЕТНЫХ ТЕКСТОВ ИЛИ ТРАКТУЮЩИХ ОПРЕДЕЛЕННЫЙ ИДЕОЛОГИЧЕСКИЙ СЮЖЕТ.

ИССЛЕДОВАНИЕ ПЛАТТА ПОДЧЕРКНУТО МЕЖДИСЦИПЛИНАРНО: АНАЛИТИЧЕСКИЙ ИНСТРУМЕНТАРИЙ АВТОР ЗАИМСТВУЕТ ИЗ ДРУГИХ НАУК - ПРЕЖДЕ ВСЕГО АНТРОПОЛОГИИ И ПСИХОЛОГИИ. КАК УЖЕ БЫЛО СКАЗАНО, ОБЪЕКТОМ ОПИСАНИЯ ПЛАТТА ЯВЛЯЕТСЯ КОЛЛЕКТИВНАЯ ИДЕНТИЧНОСТЬ, УСТОЙЧИВАЯ СТРУКТУРА КОЛЛЕКТИВНОГО БЕССОЗНАТЕЛЬНОГО, ВЫРАЖАЮЩЕГО СЕБЯ ЧЕРЕЗ КУЛЬТУРНУЮ ТРАДИЦИЮ. КЛЮЧЕВОЙ ТЕРМИН, КОТОРЫМ ОБОЗНАЧЕНА ФУНКЦИЯ ИВАНА И ПЕТРА В РУССКОЙ ИСТОРИЧЕСКОЙ МИФОЛОГИИ, - «ПОРОГОВЫЕ ФИГУРЫ» (liminal figures). В АНТРОПОЛОГИИ ЭТИМ ПОНЯТИЕМ ОБОЗНАЧАЕТСЯ ПЕРЕХОДНЫЙ НЕОПРЕДЕЛЕННЫЙ ХАРАКТЕР РИТУАЛЬНОГО ДЕЙСТВИЯ, А В СОЦИАЛЬНОЙ ПСИХОЛОГИИ ОНО ИСПОЛЬЗУЕТСЯ, ЧТО ВАЖНО, ДЛЯ УКАЗАНИЯ НА ОСОБЫЙ ХАРАКТЕР ПЕРЕЖИВАНИЙ, СВЯЗАННЫХ С ОБЩЕСТВЕННОЙ И ЛИЧНОСТНОЙ ИНИЦИАЦИЕЙ. В КНИГЕ ПЛАТТА РЕЧЬ ИДЕТ ОБ ИНИЦИАЦИИ НАРОДА ИЛИ НАЦИИ, ПЕРЕХОДА ОТ ТРАДИЦИОННОГО ОБЩЕСТВА К ИНДУСТРИАЛЬНОМУ, КОТОРЫЙ МОЖЕТ СОПРОВОЖДАТЬСЯ РАЗНОГО РОДА КОЛЛЕКТИВНЫМИ ТРАВМАМИ, ТАКИМИ, НАПРИМЕР, КАК ПОЯВЛЕНИЕ ИДЕИ ЭКОНОМИЧЕСКОГО, ПОЛИТИЧЕСКОГО ИЛИ КУЛЬТУРНОГО «ОТСТАВАНИЯ» И, ОДНОВРЕМЕННО, МЫСЛИ ОБ ОСОБОСТИ» НАЦИОНАЛЬНОГО ИСТОРИЧЕСКОГО ПУТИ, КОТОРАЯ ПОДПИТЫВАЕТСЯ АКТИВНОЙ И ЗАЧАСТУЮ УСПЕШНОЙ ИМПЕРСКОЙ ПОЛИТИКОЙ. ЭТА ВООБРАЖАЕМАЯ ИСКЛЮЧИТЕЛЬНОСТЬ ПОЛУЧАЕТ СОБСТВЕННОЕ ИСТОРИКО-КУЛЬТУРНОЕ НАПОЛНЕНИЕ - С ТОЧКИ ЗРЕНИЯ ПЛАТТА, ВАЖНЕЙШИМ ИНСТРУМЕНТОМ, ПОЗВОЛЯЮЩИМ ПРЕОДОЛЕТЬ ПСИХОЛОГИЧЕСКИЙ ДИСКОМФОРТ ПЕРЕХОДНОЙ СТАДИИ И, С ДРУГОЙ СТОРОНЫ, ДИСЦИПЛИНИРОВАТЬ ОБЩЕСТВО, ЯВЛЯЕТСЯ МИФ О НЕРАЗРЫВНОМ ЕДИНСТВЕ ВЕЛИЧИЯ И НАСИЛИЯ (В ТОМ ЧИСЛЕ СЕМЕЙНОГО), ВОПЛОЩЕННЫЙ В ФИГУРАХ ИВАНА И ПЕТРА. ТАК ПРОИСХОДИТ ПРОЦЕСС ОСОЗНАНИЯ, КОНСТРУИРОВАНИЯ И ВЫТЕСНЕНИЯ КОЛЛЕКТИВНОЙ ТРАВМЫ (ФУНДАМЕНТАЛЬНЫМ ДЛЯ РАБОТЫ ПЛАТТА ЯВЛЯЕТСЯ ЗАИМСТВОВАННЫЙ ИЗ ПСИХОЛОГИИ КОНЦЕПТ «chosen but disavowal trauma»). ОСОБЕННОСТЬЮ ЭТОГО ПРЕДСТАВЛЕНИЯ О МИРЕ ЯВЛЯЕТСЯ, ЕСЛИ МОЖНО ТАК ВЫРАЗИТЬСЯ, СВОЕОБРАЗНАЯ РЕПУТАЦИОННАЯ ИНЕРЦИЯ: ИНВАРИАНТ МИФА ВОСПРОИЗВОДИТСЯ НА КАЖДОМ НОВОМ ВИТКЕ ИДЕОЛОГИЧЕСКОЙ АКТИВНОСТИ И СТРУКТУРИРУЕТ ПОСЛЕДУЮЩУЮ РЕЦЕПЦИЮ. В ИТОГЕ, МИФОЛОГИЗИРОВАННЫЕ ОБРАЗЫ ИВАНА И ПЕТРА БОЛЬШЕ ГОВОРЯТ О СОВРЕМЕННОМ ПОЛИТИЧЕСКОМ КОНТЕКСТЕ, НЕЖЕЛИ О РОССИИ «ИСТОРИЧЕСКИХ» ИВАНА И ПЕТРА. 
НЕ ИМЕЯ ВОЗМОЖНОСТИ ПОДРОБНО РАССМОТРЕТЬ ВСЕ АНАЛИЗИРУЕМЫЕ В КНИГЕ СЮЖЕТЫ, ОСТАНОВИМСЯ НА ТЕХ, КОТОРЫЕ ПОКАЗАЛИСЬ НАМ НАИБОЛЕЕ ИНТЕРЕСНЫМИ. ТЕМУ ТРАВМЫ - НЕРАЗРЫВНОГО СОЧЕТАНИЯ ВЕЛИЧИЯ И НАСИЛИЯ, ЗАПЕЧАТЛЕННОГО В ОБРАЗАХ РУССКИХ МОНАРХОВ - ПЛАТТ ВОЗВОДИТ К 1821 Г., КОГДА В ПЕТЕРБУРГЕ ВЫХОДИТ ДЕВЯТЫЙ ТОМ «ИСТОРИИ ГОСУДАРСТВА РОССИЙСКОГО» КАРАМЗИНА. ОПИСАНИЕ ЦАРСТВОВАНИЯ ИВАНА IV ПРОИЗВЕЛО СИЛЬНЕЙШЕЕ ВПЕЧАТЛЕНИЕ НА ОБРАЗОВАННУЮ ПУБЛИКУ: ВО-ПЕРВЫХ, ИЗ-ЗА МАСШТАБА БЕДСТВИЙ, КОТОРЫМ ПОДВЕРГЛИСЬ ВСЕ БЕЗ ИСКЛЮЧЕНИЯ СТРАТЫ РУССКОГО ОБЩЕСТВА ВТОРОЙ ПОЛОВИНЫ XVI В., ВО-ВТОРЫХ, ПОТОМУ, ЧТО ГЛАВНЫМ ВИНОВНИКОМ ЗЛОСЧАСТИЙ ПОДДАННЫХ ОКАЗАЛСЯ САМ РУССКИЙ ЦАРЬ. МОТИВ ТРАВМЫ ЯВЛЯЕТСЯ ЦЕНТРАЛЬНЫМ ДЛЯ ГЛАВЫ МОНОГРАФИИ, ПОСВЯЩЕННОЙ ПЕТРОВСКОЙ ТЕМЕ В 1830-1850-Е ГГ. ПЛАТТ КОНСТАТИРУЕТ, ЧТО В ЗНАМЕНИТЫХ «СТАНСАХ» ПУШКИНА 1826 Г. НАРЯДУ С ПРИВЫЧНЫМ ВОСХВАЛЕНИЕМ ДОСТОИНСТВ ПЕТРА УПОМИНАЕТСЯ СТРЕЛЕЦКИЙ БУНТ 1698 Г., ОТЧЕТЛИВО СООТНЕСЕННЫЙ С ВОССТАНИЕМ ДЕКАБРИСТОВ. ПЕТРОВСКИЙ МИФ ДИКТУЕТ НИКОЛАЮ І СЦЕНАРИЙ ИСКУПЛЕНИЯ ПРОЛИТОЙ В НАЧАЛЕ ЦАРСТВОВАНИЯ КРОВИ - ЧЕРЕЗ ИНСТИТУТ МИЛОСТИ И ПОСТРОЕНИЕ «СВЕТЛОГО БУДУЩЕГО», ВО ИМЯ КОТОРОГО СЛЕДОВАЛО ПРИНЕСТИ ЖЕРТВУ. ПРЕДЛОЖЕННАЯ ПУШКИНЫМ МОДЕЛЬ БЫСТРО УСТАРЕЛА. КАК СЛЕДСТВИЕ, УЖЕ В «МЕДНОМ ВСАДНИКЕ» ПОЭТ СФОРМУЛИРОВАЛ НОВУЮ КОНЦЕПЦИЮ ПЕТРОВСКОГО ЦАРСТВОВАНИЯ. ВОПЛОЩАЯ ЦИВИЛИЗАЦИЮ, ПЕТР ВСТУПАЕТ В СЛОЖНЫЕ ОТНОШЕНИЯ С ПРИРОДНЫМИ СИЛАМИ И ИНДИВИДУУМОМ, ПРИЧЕМ ИТОГ КОНФЛИКТА НЕОДНОЗНАЧЕН - И НЕВЫГОДЕН ДЛЯ ЦАРЯ. ПЕТР «МЕДНОГО ВСАДНИКА» БАЛАНСИРУЕТ МЕЖДУ БОЖЕСТВЕННЫМ И ДЬЯВОЛЬСКИМ, ДЕСПОТИЗМОМ И РАЗУМНЫМ ПРАВЛЕНИЕМ, ЖИЗНЬЮ И СМЕРТЬЮ. НАСИЛИЕ ПЕТРА НАД ПРИРОДОЙ И ЧЕЛОВЕКОМ ОКАЗЫВАЕТСЯ СВОЕГО РОДА ФИГУРОЙ УМОЛЧАНИЯ, КОТОРУЮ ДЕЗАВУИРУЕТ ПУШКИН.

ВО ВТОРОЙ ПОЛОВИНЕ ХІХ В. НАИБОЛЕЕ ЯРКИЕ И ОРИГИНАЛЬНЫЕ ТРАКТОВКИ «ПЕТРОВСКО-ИВАНОВСКОГО» МИФА ДАЛИ НЕ ИСТОРИОГРАФИЯ И ЛИТЕРАТУРА, А ТЕАТР И ЖИВОПИСЬ. «ЦАРСТВОВАВШИЙ» НА СЦЕНЕ ИВАН ИНТЕРЕСОВАЛ СОВРЕМЕННИКОВ НЕ ТОЛЬКО СВОЕЙ ПОЛИТИКОЙ, НО И ИНДИВИДУАЛЬНОЙ ПСИХОЛОГИЕЙ И СПЕЦИФИЧЕСКИМ ЭМОЦИОНАЛЬНЫЙ ПОВЕДЕНИЕМ. ТАК, ПОЛОТНА «ПЕТР I ДОПРАШИВАЕТ ЦАРЕВИЧА АЛЕКСЕЯ ПЕТРОВИЧА В ПЕТЕРГОФЕ» НИКОЛАЯ ГЕ (1871) И «ИВАН ГРОЗНЫЙ И ЕГО СЫН ИВАН, 16 НОЯБРЯ 1581» ИЛЬИ РЕПИНА (1885) ВЫЗВАЛИ НЕОБЫКНОВЕННЫЙ ИНТЕРЕС У ЗРИТЕЛЕЙ. КАРТИНА ГЕ ОТЛИЧАЛАСЬ РАЦИОНАЛЬНОСТЬЮ, ХОЛОДНОСТЬЮ, ПРЕВАЛИРОВАНИЕМ ИСТОРИЧЕСКОГО ЭЛЕМЕНТА НАД ТЕАТРАЛЬНЫМ; РЕПИН, НАПРОТИВ, ИЗОБРАЗИЛ ОТЦА И СЫНА В РАДИКАЛЬНО ИНОЙ СИТУАЦИИ, ВЫВЕЛ НА ПЕРВЫЙ ПЛАН ЭМОЦИОНАЛЬНУЮ СОСТАВЛЯЮЩУЮ СЫНОУБИЙСТВА. КАРТИНУ РЕПИНА, ПО МНЕНИЮ ПЛАТТА, МОЖНО СЧИТАТЬ СВОЕОБРАЗНЫМ ОТВЕТОМ НА ПОЛОТНО ГЕ. ОСНОВНОЙ ИДЕОЛОГИЧЕСКИЙ СМЫСЛ ПЕРЕКЛИЧКИ СОСТОИТ В СТОЛКНОВЕНИИ ДВУХ КОНЦЕПЦИЙ РОССИИ - КАК НАСЛЕДНИЦЫ ПЕТРОВСКИХ ПРЕОБРАЗОВАНИЙ И КАК ТРАДИЦИОННОЙ ВОСТОЧНОЙ МОНАРХИИ: В ТОТ МОМЕНТ, КОГДА РОССИЯ СТРЕМИТСЯ СТАТЬ ЗАПАДНОЙ СТРАНОЙ, РЕЛЬЕФНЕЕ ПРОСТУПАЕТ ЕЕ ВОСТОЧНАЯ ПРИРОДА, А СТРЕМЛЕНИЕ К «РАЗУМНЫМ» РЕФОРМАМ ОБОРАЧИВАЕТСЯ НАСИЛИЕМ И ТРАГЕДИЕЙ.

6 ЕСЛИ ВО ВТОРОЙ ПОЛОВИНЕ ХІХ В. ПОЛИТИЧЕСКИМ ФОНОМ ПРИ ОБСУЖДЕНИИ ВОПРОСА О СИМВОЛИЧЕСКОМ ЗНАЧЕНИИ РУССКИХ МОНАРХОВ БЫЛИ ВЕЛИКИЕ РЕФОРМЫ, ТО В НАЧАЛЕ ХХ В. МИФОЛОГИЯ ИВАНА ГРОЗНОГО И ПЕТРА ВЕЛИКОГО 
ОКАЗЫВАЕТСЯ ВПИСАНА В НОВЫЙ ИДЕОЛОГИЧЕСКИЙ КОНТЕКСТ. ПО МНЕНИЮ ПЛАТТА, ИСТОРИЧЕСКОЕ СОЗНАНИЕ ЭПОХИ БЫЛО ОХВАЧЕНО ОЖИДАНИЕМ СОЦИАЛЬНОГО КАТАКЛИЗМА И ГИБЕЛИ РОССИЙСКОЙ ИМПЕРИИ. В ЭТОЙ СИТУАЦИИ ФИГУРЫ ИВАНА ГРОЗНОГО И ПЕТРА ВЕЛИКОГО ПРИОБРЕЛИ НЕСОМНЕННУЮ АКТУАЛЬНОСТЬ КАК ЭСХАТОЛОГИЧЕСКИЕ ПРОВОЗВЕСТНИКИ БУДУЩЕЙ СУДЬБЫ РОССИИ. ПЛАТТ ПОДРОБНО АНАЛИЗИРУЕТ РОМАН Д.С. МЕРЕЖКОВСКОГО «АНТИХРИСТ (ПЕТР И АЛЕКСЕЙ)» (1904), ОПИСЫВАЯ СЛОЖНУЮ СТРУКТУРУ ОБРАЗОВ ПЕТРА И АЛЕКСЕЯ КАК «ПОРОГОВЫХ» И ДИАЛЕКТИЧЕСКИХ ФИГУР. ДАЛЕЕ, АВТОР МОНОГРАФИИ РАЗБИРАЕТ ТРЕХТОМНЫЙ ТРУД П.А. МИЛЮКОВА «ОЧЕРКИ ИСТОРИИ РУССКОЙ КУЛЬТУРЫ» (1896-1903). У МИЛЮКОВА ИВАН И ПЕТР СТАНОВЯТСЯ СИМВОЛАМИ ГЛУБИННОГО, СВОЙСТВЕННОГО РОССИИ ПРОТИВОРЕЧИЯ МЕЖДУ ЦЕЛЯМИ И СРЕДСТВАМИ: ПРОГРЕССИВНЫЕ РЕФОРМЫ ПРОВОДЯТСЯ С ПОМОЩЬЮ АРХАИЧЕСКИХ НАСИЛЬСТВЕННЫХ МЕТОДОВ. СОПЕРНИЧЕСТВО РАЗНЫХ ПОЛИТИЧЕСКИХ ТЕНДЕНЦИЙ В РУССКОЙ ИСТОРИИ ПРОЕЦИРОВАЛОСЬ МИЛЮКОВЫМ НА СОВРЕМЕННОСТЬ: ОЖИДАНИЕ НОВЫХ СОЦИАЛЬНЫХ ТРАНСФОРМАЦИЙ И РАЗРУШЕНИЯ СУЩЕСТВУЮЩЕГО МИРОПОРЯДКА СБЛИЖАЛО ЕГО ПОЗИЦИЮ С МИСТИЧЕСКИМИ ОТКРОВЕНИЯМИ МЕРЕЖКОВСКОГО.

ПРЕДСКАЗАНИЯ ПРЕДРЕВОЛЮЦИОННЫХ ВИЗИОНЕРОВ, КАК ИЗВЕСТНО, С ЛИХВОЙ СБЫЛИСЬ. В РОССИИ ВОЗНИК НОВЫЙ ПОРЯДОК, ДЕМОНСТРАТИВНО ОТМЕЖЕВАВШИЙСЯ ОТ ИМПЕРАТОРСКОГО ПРОШЛОГО. В 1920-Е И ОСОБЕННО В 1930-Е ГГ. ФОРМИРУЕТСЯ ТОТАЛИТАРНАЯ ИДЕОЛОГИЯ, КОТОРАЯ, ВПРОЧЕМ, НЕ ПРЕНЕБРЕГАЕТ СИМВОЛИЧЕСКИМ ПОТЕНЦИАЛОМ РУССКИХ МОНАРХОВ, А ПЫТАЕТСЯ ПЕРЕКОДИРОВАТЬ ИХ ОБРАЗЫ И ПОСТАВИТЬ ИХ НА СЛУЖБУ НОВОМУ РЕЖИМУ. ВМЕСТЕ С ТЕМ, ИСТОРИЧЕСКИЕ МЕТАСЮЖЕТЫ ЧАСТО НЕ ПОДДАЮТСЯ МАНИПУЛЯЦИИ, А, НАОБОРОТ, САМИ ЗАДАЮТ ПАРАМЕТРЫ ДЛЯ ИДЕОЛОГИЧЕСКИХ ПОСТРОЕНИЙ СОВЕТСКОЙ ЭПОХИ. В 1930-Х ГГ. ИВАН И ПЕТР ВОЗВРАЩАЮТСЯ В ОФИЦИАЛЬНЫЙ ЗАЛ СЛАВЫ РУССКОГО-СОВЕТСКОГО ГОСУДАРСТВА (НАПРИМЕР, В «КРАТКОМ КУРСЕ ИСТОРИИ СССР» А. ШЕСТАКОВА 1937 Г.) КАК ПРАВИТЕЛИ, СПОСОБНЫЕ - ПОДОБНО СОВЕТСКОЙ ПОЛИТИЧЕСКОЙ ЭЛИТЕ ЖЕРТВОВАТЬ ИНДИВИДУАЛЬНЫМ, В ТОМ ЧИСЛЕ ЧЕЛОВЕЧЕСКОЙ ЖИЗНЬЮ, РАДИ БЛАГОДЕНСТВИЯ ОБЩЕСТВА В ЦЕЛОМ.

МЕХАНИЗМЫ ПРЕОБРАЗОВАНИЯ ИСТОРИЧЕСКОЙ ТРАВМЫ В КОЛЛЕКТИВНУЮ НАДЕЖДУ НА БУДУЩЕЕ ИССЛЕДУЮТСЯ ПЛАТТОМ НА ПРИМЕРЕ НЕЗАКОНЧЕННОЙ КИНОТРИЛОГИИ С. ЭЙЗЕНШТЕЙНА «ИВАН ГРОЗНЫЙ». СТАЛИНСКОЕ КИНО ПРЕЖДЕ ВСЕГО СТРЕМИЛОСЬ ОБЕЗДВИЖИТЬ ИСТОРИЧЕСКОЕ ВРЕМЯ, ПОДЧЕРКНУТЬ ЕГО ЦИКЛИЧНОСТЬ (А ВНУТРИ ЭТОЙ ЦИКЛИЧНОСТИ ИДЕНТИФИЦИРОВАТЬ УСТОЙЧИВУЮ ФИГУРУ ВНЕШНЕГО ВРАГА: ИВАН ГРОЗНЫЙ ИЛИ ПЕТР ВЕЛИКИЙ ПОВТОРЯЛИСЬ В СТАЛИНЕ, ТЕВТОНСКИЕ РЫЦАРИ В НАЦИСТАХ И Т.Д.). С ОДНОЙ СТОРОНЫ, ИВАН У ЭЙЗЕНШТЕЙНА СИМВОЛИЗИРУЕТ СОБОЙ ОДИН ИЗ ВИТКОВ ГЕРОИЧЕСКОЙ БОРЬБЫ ЗА СОЦИАЛЬНЫЙ ПРОГРЕСС, ТРЕБУЮЩЕЙ ЖЕСТКИХ РЕПРЕССИЙ В ОТНОШЕНИИ ВНЕШНЕГО И ВНУТРЕННЕГО ВРАГА. С ДРУГОЙ, ПО МНЕНИЮ ПЛАТТА, ЗЛОДЕЯНИЯ, СОВЕРШЕННЫЕ ИВАНОМ В ФИЛЬМЕ ЭЙЗЕНШТЕЙНА, СВИДЕТЕЛЬСТВУЮТ И О МОРАЛЬНОМ ПОРАЖЕНИИ ЖЕСТОКОГО ЦАРЯ. ТАКИМ ОБРАЗОМ, В ФИЛЬМЕ ВНОВЬ ПРОЯВЛЯЕТСЯ БАЗОВАЯ АМБИВАЛЕНТНОСТЬ КУЛЬТУРНОГО МИФА О ВЕЛИЧИИ И НАСИЛИИ, ОБ ОСОЗНАННОЙ ТРАВМЕ, СВЯЗАННОЙ С ФИГУРАМИ ИВАНА И ПЕТРА, КОТОРУЮ АЛЛЕГОРИЧЕСКИ ОБЫГРЫВАЕТ РЕЖИССЕР.

БЛИЖАЙШИЙ ИСТОРИОГРАФИЧЕСКИЙ КОНТЕКСТ ИССЛЕДОВАНИЯ ПЛАТТА СОСТАВЛЯЕТ КНИГА Ф.Б. ШЕНКА «АЛЕКСАНДР НЕВСКИЙ В РУССКОЙ КУЛЬТУРНОЙ ПАМЯТИ» (2007). СРАВНЕНИЕ ДВУХ МОНОГРАФИЙ ПОЗВОЛЯЕТ ЯСНЕЕ УВИДЕТЬ ОСОБЕННОСТИ ПОДХОДА 
АМЕРИКАНСКОГО ИССЛЕДОВАТЕЛЯ. ТАК, ЕСЛИ ШЕНК СТРЕМИТСЯ ОХАРАКТЕРИЗОВАТЬ ВСЕ ЭТАПЫ РАЗВИТИЯ МИФОЛОГИЧЕСКОЙ ИСТОРИИ ИЗБРАННОГО ИМ РУССКОГО КНЯЗЯ, ТО ПЛАТТ ОТКАЗЫВАЕТСЯ ОТ ТОТАЛЬНОГО ОПИСАНИЯ, СОСРЕДОТОЧИВАЯСЬ НА «БОЛЬШИХ» ТЕКСТАХ, УДЕЛЬНЫЙ ВЕС КОТОРЫХ В КУЛЬТУРЕ ПРИЗВАН ОПРАВДАТЬ СТРАТЕГИЮ УЧЕНОГО. ОБА ИССЛЕДОВАТЕЛЯ СХОДЯТСЯ В ТОМ, ЧТО НАЦИОНАЛЬНЫЙ ГОСУДАРСТВЕННЫЙ МИФ НЕ ВОЗНИКАЕТ ex-nihilo, НО ЯВЛЯЕТСЯ, ПОЛЬЗУЯСЬ ВЫРАЖЕНИЕМ ШЕНКА, «ПЕРЕОСМЫСЛЕНИЕМ УЖЕ ИМЕВШИХСЯ ПОВЕСТВОВАНИЙ И КОЛЛАЖАМИ ИЗ НИХ, Т.Е. ПРОДУКТАМИ СВОЕОБРАЗНОГО “МЕЖКУЛЬТУРНОГО БРИКОЛАЖА"»33. ОДНАКО ОБЩИЙ МЕТОДОЛОГИЧЕСКИЙ ПОСЫЛ ПРИВОДИТ АВТОРОВ К ДВУМ СОВЕРШЕННО РАЗНЫМ АНАЛИТИЧЕСКИМ СТРАТЕГИЯМ: ЕСЛИ ШЕНК ПОСЛЕДОВАТЕЛЬНО ИССЛЕДУЕТ РАЗВОРАЧИВАНИЕ МИФА ОБ АЛЕКСАНДРЕ НЕВСКОМ НАЧИНАЯ С 1263 Г., ТО ИСТОРИЯ ПЛАТТА (С НЕЗНАЧИТЕЛЬНЫМИ ОГОВОРКАМИ) НАЧИНАЕТСЯ ЛИШЬ В ПЕРВОЙ ЧЕТВЕРТИ ХІХ В. ЧТО ТЕРЯЕТ МОНОГРАФИЯ ПЛАТТА В РЕЗУЛЬТАТЕ ТАКОГО ВЫБОРА?

ВО-ПЕРВЫХ, К «ПОТЕРЯМ» МОЖНО ОТНЕСТИ ОТСУТСТВИЕ СВЕДЕНИЙ О ФУНКЦИИ ИВАНА ГРОЗНОГО В ИДЕОЛОГИЧЕСКИХ ПОСТРОЕНИЯХ САМОГО ПЕТРА І. КАК ПОКАЗАЛА ЕЛЕНА ПОГОСЯН, ПЕТР ПРЕДПОЧИТАЛ СЧИТАТЬ ОСНОВАТЕЛЕМ РОССИЙСКОЙ ГОСУДАРСТВЕННОСТИ НЕ ИВАНА, А ЕГО ОТЦА, ВАСИЛИЯ III, СЫНА ВИЗАНТИЙСКОЙ ЦАРЕВНЫ СОФЬИ ПАЛЕОЛОГ. ТАКАЯ ИНТЕРПРЕТАЦИЯ ОТЕЧЕСТВЕННОЙ ИСТОРИИ БЫЛА МОТИВИРОВАНА ВАЖНЕЙШИМИ ДЛЯ ПЕТРА СООБРАЖЕНИЯМИ ОБ ИМПЕРСКОМ НАСЛЕДОВАНИИ (РОССИЯ КАК НАСЛЕДНИЦА ВИЗАНТИИ, ПРИЗНАННАЯ ВЕЛИКОЙ РИМСКОЙ ИМПЕРИЕЙ ГЕРМАНСКОЙ НАЦИИ $)^{4}$. ЭТА МИФОЛОГИЯ ОКАЗАЛА ВАЖНОЕ ВЛИЯНИЕ НА ИДЕОЛОГИЧЕСКИЕ КОНСТРУКТЫ, СОЗДАННЫЕ В РОССИИ XVIII В., В ЧАСТНОСТИ ДЛЯ ОБЪЯСНЕНИЯ ПРЕТЕНЗИЙ РОССИИ НА КОНСТАНТИНОПОЛЬ. ВО-ВТОРЫХ, ИЗ ПОЛЯ ЗРЕНИЯ ПЛАТТА СОВЕРШЕННО ВЫПАЛА СТОЛЬ ЗНАЧИМАЯ ДЛЯ ПРОБЛЕМАТИКИ «ПОРОГОВОСТИ» ТЕМА КАК ОППОЗИЦИЯ ПЕТРА-ВАРВАРА ПЕТРУЦИВИЛИЗОВАННОМУ ПРАВИТЕЛЮ (А ТАКЖЕ УКОРЕНЕННЫЙ В ЭТОМ ПРОТИВОПОСТАВЛЕНИИ КОЛОССАЛЬНЫЙ ПЛАСТ СМЫСЛОВ И ЗНАЧЕНИЙ, СВЯЗАННЫЙ С «ВЕЛИЧИЕМ», «НАСИЛИЕМ» И Т.Д.). СЛЕДУЕТ ПОДЧЕРКНУТЬ, ЧТО В ФОРМАЛЬНЫХ ОСНОВАНИЯХ ОБРАТИТЬСЯ К ЭПОХЕ ДО ХІХ В. НЕТ НЕДОСТАТКА: СОПОСТАВЛЕНИЯ ПЕТРА И ИВАНА ВСТРЕЧАЮТСЯ ВО ФРАНЦУЗСКОЙ РОССИКЕ УЖЕ ВО ВТОРОЙ ПОЛОВИНЕ XVIII СТОЛЕТИЯ 5 . ИГНОРИРОВАНИЕ ЭТОГО ПЕРИОДА ОБЕДНЯЕТ ИССЛЕДОВАНИЕ ПЛАТТА И МЕТОДОЛОГИЧЕСКИ - ОНО ВЫГЛЯДИТ ИЗЛИШНЕ «РУССО-ЦЕНТРИЧНЫМ», ПОЛНОСТЬЮ ЛИШЕННЫМ СРАВНИТЕЛЬНОЙ ПЕРСПЕКТИВЫ. МЕЖДУ ТЕМ, ОБРАЗ ПЕТРА В ХІХ В. СЛОЖНО РАССМАТРИВАТЬ ВНЕ КОНТЕКСТА ФРАНЦУЗСКОЙ ПУБЛИЦИСТИКИ XVIII В., А ИДЕОЛОГИЯ «ОСОБОСТИ» НЕМЫСЛИМА ВНЕ ФАКТОВ ГЕРМАНСКОГО ВЛИЯНИЯ. В-ТРЕТЬИХ, В ИССЛЕДОВАНИИ ПЛАТТА ОТСУТСТВУЕТ АНАЛИЗ ЛИТЕРАТУРНОЙ, ИСТОРИОГРАФИЧЕСКОЙ И ТЕАТРАЛЬНОЙ ПРОДУКЦИИ САМОГО НАЧАЛА ХІХ В. - ЭПОХИ НЕОБЫЧАЙНОЙ МИФОТВОРЧЕСКОЙ АКТИВНОСТИ РУССКИХ ИНТЕЛЛЕКТУАЛОВ, УВЕНЧАВШЕЙСЯ СОБЫТИЯМИ 1812-1814 ГГ. ВОЙНА С НАПОЛЕОНОМ, КАК ИЗВЕСТНО, ОКАЗАЛА ГЛУБОКОЕ ВЛИЯНИЕ НА ПРЕДСТАВЛЕНИЯ РУССКИХ О СЕБЕ И СВОЕМ МЕСТЕ В ЕВРОПЕ. НЕ В ПОСЛЕДНЮЮ ОЧЕРЕДЬ, ОДНИМ ИЗ ЕЕ РЕЗУЛЬТАТОВ СТАЛО ИНТЕНСИВНОЕ ОСМЫСЛЕНИЕ ОБРАЗОВ ПЕТРА ВЕЛИКОГО И ИВАНА ГРОЗНОГО, И ВОПРОСА ОБ ИХ МЕСТЕ В ПАНТЕОНЕ НАЦИОНАЛЬНЫХ ГЕРОЕВ. ИГНОРИРУЯ ЭТУ ЭПОХУ, ПЛАТТ СОЗДАЕТ У ЧИТАТЕЛЯ ЛОЖНОЕ ВПЕЧАТЛЕНИЕ, ЧТО ДО КАРАМЗИНА НИКАКИХ ВАЖНЫХ ДЕБАТОВ О 
РУССКИХ МОНАРХАХ НЕ ВЕЛОСЬ И ЧТО ПРЕДСТАВЛЕНИЯ ОБ ИВАНЕ И ПЕТРЕ ПОЯВЛЯЮТСЯ ЕДВА ЛИ НЕ «ИЗ ПУСТОТЫ».

В ЧЕМ ПРИЧИНА ПОЯВЛЕНИЯ УКАЗАННЫХ ЛАКУН? КАК ПРЕДСТАВЛЯЕТСЯ, ОНИ СВЯЗАНЫ С МЕТОДОЛОГИЧЕСКОЙ ПОЗИЦИЕЙ ИССЛЕДОВАТЕЛЯ. КАК БЫЛО ОТМЕЧЕНО ВЫШЕ, В XVIII-ПЕРВОЙ ПОЛОВИНЕ ХІХ ВВ. РЕПУТАЦИИ ЦАРЕЙ ПЕТРА И ИВАНА БЫЛИ РЕШИТЕЛЬНО НЕСОПОСТАВИМЫ: РОЛЬ ПЕТРА В ПОСТРОЕНИИ НАЦИОНАЛЬНОЙ ИДЕНТИЧНОСТИ ОКАЗЫВАЛАСЬ НЕИЗМЕРИМО ВАЖНЕЕ ЗАСЛУГ ЕГО ПРЕДШЕСТВЕННИКА ИЗ ДИНАСТИИ РЮРИКОВИЧЕЙ. ИСТОРИЧЕСКИЙ КОНТЕКСТ КУЛЬТУРНОГО РОДСТВА ИВАНА И ПЕТРА ДЛЯ ПЛАТТА СУЩЕСТВЕННО ВАЖНЕЕ РАСХОЖДЕНИЙ МЕЖДУ ИХ РЕПУТАЦИЯМИ - КАК СЛЕДСТВИЕ, ПЛАТТ ПРИСТУПАЕТ К АНАЛИЗУ ПЕТРОВСКОЙ МИФОЛОГИИ В ТОТ МОМЕНТ, КОГДА В РУССКОЙ КУЛЬТУРЕ АКТУАЛИЗИРУЕТСЯ ОБРАЗ ИВАНА ГРОЗНОГО, Т.Е. В СЕРЕДИНЕ ХІХ В. ПАРАЛЛЕЛЬ МЕЖДУ ИВАНОМ И ПЕТРОМ, СТРОГО ГОВОРЯ, БАЗИРУЕТСЯ НА ДВУХ КРИТЕРИЯХ: ВО-ПЕРВЫХ, РОЛИ В ПРОЦЕССЕ ГОСУДАРСТВЕННОГО СТРОИТЕЛЬСТВА, ВО-ВТОРЫХ, ФУНКЦИИ В МАГИСТРАЛЬНЫХ «ПСИХОЛОГИЧЕСКИХ СЮЖЕТАХ» РУССКОЙ КУЛЬТУРНОЙ ИСТОРИИ (В СВЯЗИ С ПРИЧИНАМИ И ПОСЛЕДСТВИЯМИ СЫНОУБИЙСТВА). ВНОВЬ ОБА КРИТЕРИЯ КАЖУТСЯ РЕЛЕВАНТНЫМИ ИМЕННО ДЛЯ ВТОРОЙ ПОЛОВИНЫ ХІХ-ХХ ВЕКА, КОГДА РЕЗКО РАСШИРИЛОСЬ ПРОСТРАНСТВО ПУБЛИЧНОГО ОБСУЖДЕНИЯ СТРАТЕГИИ ГОСУДАРСТВЕННОГО РАЗВИТИЯ РОССИИ, А ПСИХОЛОГИЧЕСКАЯ НАУКА СДЕЛАЛА НЕСКОЛЬКО РЕШИТЕЛЬНЫХ ШАГОВ ВПЕРЕД. ПОЖАЛУЙ, НАИБОЛЬШИЕ СОМНЕНИЯ ВЫЗЫВАЕТ ПЕРЕНЕСЕНИЕ ПЛАТТОМ МЕТОДОВ И ИНСТРУМЕНТАРИЯ ДРУГИХ НАУК В ИСТОРИЮ КУЛЬТУРЫ. КАК ПРИЗНАЛ САМ АВТОР В ХОДЕ ОБСУЖДЕНИЯ ЕГО ДОКЛАДА ОБ ИВАНЕ И ПЕТРЕ НА ХІХ-ЫХ БАННЫХ ЧТЕНИЯХ В МОСКВЕ (1-2 АПРЕЛЯ 2011 Г.), «СУЩЕСТВУЕТ ОПРЕДЕЛЕННАЯ СЛОЖНОСТЬ В САМОМ ПЕРЕНЕСЕНИИ ПСИХОАНАЛИТИЧЕСКОЙ ТЕРМИНОЛОГИИ С ИНДИВИДУУМА НА ОБЩЕСТВО: ТАК, ДЕЗАВУИРОВАНИЕ МОЖЕТ ВОСПРИНИМАТЬСЯ СКОРЕЕ КАК МЕТАФОРА, ЧЕМ КАК РЕАЛЬНЫЙ ПСИХИЧЕСКИЙ ПРОЦЕСС, ПОЭТОМУ ОДНОЗНАЧНОГО СООТВЕТСТВИЯ С ПСИХОАНАЛИТИЧЕСКОЙ ЛОГИКОЙ ЗДЕСЬ БЫТЬ НЕ МОЖЕТ» ДЕЙСТВИТЕЛЬНО, В МОНОГРАФИИ ПЛАТТА СУЩЕСТВУЕТ ОПРЕДЕЛЕННАЯ ДИСТАНЦИЯ МЕЖДУ ВЗГЛЯДАМИ НА ПРЕДМЕТ ИНТЕЛЛЕКТУАЛОВ РАЗЛИЧНЫХ ЭПОХ И (ПСИХО)АНАЛИТИЧЕСКИМИ КАТЕГОРИЯМИ, КОТОРЫЕ ИСПОЛЬЗУЕТ ДЛЯ ИХ ОПИСАНИЯ ИССЛЕДОВАТЕЛЬ. САМА ПО СЕБЕ ТАКАЯ ДИСТАНЦИЯ ВПОЛНЕ ЕСТЕСТВЕННА, ДРУГОЕ ДЕЛО, ЧТО ОНА ДОЛЖНА БЫТЬ ОТРЕФЛЕКТИРОВАНА И ОБЪЯСНЕНА: ИНАЧЕ НАУЧНАЯ МОНОГРАФИЯ САМА РИСКУЕТ СТАТЬ ИСТОЧНИКОМ МИФА. ВПРОЧЕМ, ВЫШЕИЗЛОЖЕННЫЕ СООБРАЖЕНИЯ ОТНОСЯТСЯ К ИЗВЕСТНОМУ ЖАНРУ «КАК БЫ Я НАПИСАЛ КНИГУ, КОТОРУЮ НИКОГДА НЕ НАПИШУ» И НИ В КОЕМ СЛУЧАЕ НЕ ПРЕПЯТСТВУЮТ ИЗЪЯВЛЕНИЮ ЖИВЕЙШЕЙ ЧИТАТЕЛЬСКОЙ БЛАГОДАРНОСТИ КЕВИНУ ПЛАТТУ ЗА ПЛОДОТВОРНОЕ И УВЛЕКАТЕЛЬНОЕ ИССЛЕДОВАНИЕ. 


\section{NOTES}

1. Д.Л. МОРДОВЦЕВ, «ТРИ ДЕТОУБИЙСТВА: ИСТОРИЧЕСКАЯ ПАРАЛЛЕЛЬ», ДРЕВНЯЯ И НОВАЯ РОССИЯ, № 2, 1879, с. 135-138.

2. TAM ЖЕ, C. 138.

3. Ф.Б. ШЕНК, АЛЕКСАНДР НЕВСКИЙ В РУССКОЙ КУЛЬТУРНОЙ ПАМЯТИ: СВЯТОЙ, ПРАВИТЕЛЬ, НАЦИОНАЛЬНЫЙ ГЕРОЙ (1263-2000), М., 2007, с. 13.

4. ПОДРОБНЕЕ СМ.: Е.А. ПОГОСЯН, ПЕТР I - АРХИТЕКТОР РОССИЙСКОЙ ИСТОРИИ, СПБ., 2001, С. 199-206.

5. НАПРИМЕР, В ЧЕТВЕРТОМ ТОМЕ «ИСТОРИИ РОССИИ» П.-Ш. ЛЕВЕКА (1782). СМ.: С.А. МЕЗИН, ВЗГЛЯД ИЗ ЕВРОПЫ: ФРАНЦУЗСКИЕ АВТОРЫ ХVIII ВЕКА О ПЕТРЕ І, САРАТОВ, 2003, С. 216.

6. РЕПЛИКА К. ПЛАТТА, СОГЛАСНО ОТЧЕТУ О КОНФЕРЕНЦИИ В. ИВАНОВА: http:// magazines.russ.ru/nlo/2011/111/iv47.html. В СКОБКАХ ЗАМЕТИМ, ЧТО ВО ВВЕДЕНИИ К ИССЛЕДОВАНИЮ ПЛАТТА НАПРАШИВАЕТСЯ ССЫЛКА НА СТАТЬЮ В.М. ЖИВОВА «ИВАН СУСАНИН И ПЕТР ВЕЛИКИЙ. О КОНСТАНТАХ И ПЕРЕМЕННЫХ В СОСТАВЕ ИСТОРИЧЕСКИХ ПЕРСОНАЖЕЙ», НОВОЕ ЛИТЕРАТУРНОЕ ОБОЗРЕНИЕ, № 38 (1999), В КОТОРОЙ АВТОР, КРОМЕ ТОГО, ЧТО ВЫСКАЗЫВАЕТ ВАЖНЫЕ И ИНТЕРЕСНЫЕ МЫСЛИ О ПРИРОДЕ ПРОЦЕССА ИДЕОЛОГИЧЕСКОЙ КОНСТРУКЦИИ, ТАКЖЕ ЭКСПЛУАТИРУЕТ ПСИХОЛОГИЧЕСКИЕ ОБЪЯСНЕНИЯ СТРУКТУР КОЛЛЕКТИВНОЙ КУЛЬТУРНОЙ ПАМЯТИ - В ЧАСТНОСТИ, О «ФАЛЛИЧЕСКОЙ ПРИРОДЕ ВЛАСТИ», «СИМВОЛИЧЕСКОЙ КАСТРАЦИИ» И «ЭДИПОВОМ КОМПЛЕКСЕ». 